\title{
ON THE NUMBER OF PRIMITIVE PYTHAGOREAN QUINTUPLES
}

\section{WERNER HÜRLIMANN}

Feldstrasse 145

CH-8004, Zürich

Switzerland

e-mail: whurlimann@bluewin.ch

\begin{abstract}
We express the number of distinct primitive Pythagorean quintuples in terms of the total number of primitive representations of a square as a sum of four squares (counting zeros, permutations, and sign changes), two twisted Euler functions with Dirichlet characters of period four and eight, and three counting formulas for binary sums of squares.
\end{abstract}

\section{Introduction}

The representation of integers by sums of squares has attracted the attention of many mathematicians from Diophantus, Euler, Lagrange, to Gauss and many others. One of the oldest classes of such representations concern Pythagorean triples. More generally, for fixed $n \geq 3$, Pythagorean $n$-tuples concern the representations of squares by sums of $n-1$ nonzero squares. In dimensions $n \geq 5$ counting formulas for them do not seem to exist. Moreover, it is only recently that Hürlimann [5] has derived an exact and an asymptotic formula for the number of primitive Pythagorean quadruples.

2010 Mathematics Subject Classification: 11E25, 11A25, 11B34, 11D45.

Keywords and phrases: Diophantine equation, sum of squares, ternary quadratic form, arithmetic function, twisted Euler function.

Received March 17, 2015

(C) 2015 Scientific Advances Publishers 
A primitive Pythagorean quintuple is described by a solution in non-zero natural numbers $w, x, y, z, t$ of the Diophantine equation $w^{2}+x^{2}+y^{2}+z^{2}=t^{2}$ satisfying the condition $\operatorname{gcd}(w, x, y, z, t)=1$. Generalizing the counting mechanism in Hürlimann [5], we derive an exact counting formula for the number of primitive Pythagorean quintuples. A more detailed account of the content follows.

Section 2 summarizes some basic facts about the representation of numbers by sums of squares and more generally by quadratic forms. Usually, a counting formula for the number of representations by a quadratic form includes zeros, permutations, and sign changes. If one counts primitive representations only, then Möbius inversion of such a counting formula must be performed. To count Pythagorean $n$-tuples, it is further necessary to disregard from counting zeros. The algebraic mechanism for this is explained and a basic reduction formula to count the number of primitive quintuples is derived in Equation (2.8). The Sections 3 and 4 determine counting formulas for involved ternary and binary squares representations. The main formula for the number of distinct primitive Pythagorean quintuples is obtained in Theorem 5.1 and illustrated in the Tables 5.1 and 5.2.

\section{The Basic Algebraic Counting Mechanism}

In classical arithmetic, one is interested in representations of a number $m$ as a sum of $k \geq 2$ squares such that $x_{1}^{2}+x_{2}^{2}+\cdots+x_{k}^{2}=m$. The number of such representations, counting zeros, permutations, and sign changes, is denoted by $r_{k}(m)$. Similarly, the abbreviation $R_{k}(m)$ denotes the number of primitive representations of $m$ as a sum of $k \geq 2$ squares counting zeros, permutations, and sign changes. Given a formula for $r_{k}(m)$, one obtains from it a formula for $R_{k}(m)$ through Möbius inversion of the basic identity (e.g., Grosswald [6], Theorem 1, Subsection 1.1) 


$$
r_{k}(m)=\sum_{d^{2} \mid m} R_{k}\left(\frac{m}{d^{2}}\right) .
$$

This technique has been largely exploited by Cooper and Hirschhorn [2], who obtain a wide variety of formulas for $R_{k}(m)$ including the range $2 \leq k \leq 8$ for any $m$, and the range $9 \leq k \leq 12$ for certain values of $m$. The number of distinct primitive representations of $m$ as a sum of $k \geq 2$ non-zero squares such that $x_{1}^{2}+x_{2}^{2}+\cdots+x_{k}^{2}=m$ with $\prod_{j=1}^{k} x_{j} \neq 0$, which is denoted by $R_{k}^{d}(m)$, has not been studied very often. It is only recently that Hürlimann [5], Lemma 4, has derived a formula for $R_{3}^{d}\left(t^{2}\right)$, the number of primitive Pythagorean quadruples.

Möbius inversion is not restricted to sums of squares. Given a quadratic form $Q\left(x_{1}, x_{2}, \ldots, x_{k}\right)$, one is in general interested in integer solutions of the Diophantine equation $Q\left(x_{1}, x_{2}, \ldots, x_{k}\right)=m$ for given $m$. Then, if one denotes by $r_{Q}(m)$ the total number of solutions, and by $R_{Q}(m)$ the total number of primitive solutions, one has the generalized formula

$$
r_{Q}(m)=\sum_{d^{2} \mid m} R_{Q}\left(\frac{m}{d^{2}}\right) .
$$

Möbius inversion of (2.2) yields the formula (Cooper and Hirschhorn [2], Equation (1.3))

$$
\begin{aligned}
R_{Q}(m)= & r_{Q}(m)-\sum_{p_{1}^{2} \mid m} r_{Q}\left(\frac{m}{p_{1}^{2}}\right)+\sum_{p_{1}^{2}, p_{2}^{2} \mid m} r_{Q}\left(\frac{m}{p_{1}^{2} p_{2}^{2}}\right) \\
& -\sum_{p_{1}^{2}, p_{2}^{2}, p_{3}^{2} \mid m} r_{Q}\left(\frac{m}{p_{1}^{2} p_{2}^{2} p_{3}^{2}}\right)+\cdots+(-1)^{n} \sum_{p_{1}^{2}, \ldots, p_{n}^{2} \mid m} r_{Q}\left(\frac{m}{p_{1}^{2} \ldots p_{n}^{2}}\right)
\end{aligned}
$$

where $p_{1}, p_{2}, \ldots, p_{n}$ are the distinct primes whose squares divide $m$. 
Let us return to the topic of the present contribution. Bachet (15811638) conjectured in 1621 that every natural number can be written as a sum of four squares. The first complete proof of this statement is due to Lagrange [7]. Some further historical development and concrete expressions for $r_{4}(m)$, respectively, $R_{4}(m)$, are found in Cooper and Hirschhorn [2] (the formulas are found in Lemma 3, respectively, Theorem 1). An exact formula for the number $R_{4}^{d}\left(t^{2}\right)$ of primitive Pythagorean quintuples has not been derived so far. As will be proved later, there exist primitive Pythagorean quintuples if, and only if, the number $t>3$ is odd or $t \geq 2$ is exactly divisible by 2 . Therefore, Pythagorean quintuples for even numbers $t \geq 4$ divisible by 4 are always multiples of primitive ones.

Let us explain the basic mechanism used to derive a formula for $R_{4}^{d}\left(t^{2}\right)$. Consider the $R_{4}\left(t^{2}\right)$ integer quadruples $(w, x, y, z)$ solving $w^{2}+x^{2}+y^{2}+z^{2}=t^{2}$ taking into account zeros, permutations, and sign changes. The solutions are said to be primitive if these quadruples satisfy the condition $\operatorname{gcd}(w, x, y, z, t)=1$ in the sense that any integer is a divisor of zero, a convention made throughout. Looking at possible zeros and equal entries the distinct primitive solutions can take 7 different forms, namely,

$(w, x, y, z),(w, x, y, y),(w, x, x, x),(w, w, y, y),(0, x, y, z),(0, x, x, z)$,

and $(0,0, y, z)$,

with distinct entries $w, x, y, z \neq 0$. For each form, one must determine the number of resulting representations counting permutations and sign changes, as well as the number of distinct primitive solutions generated by this form. The latter counting function is denoted by $D_{\bullet}\left(t^{2}\right)$, where stands for the quadratic form type of the corresponding Diophantine equation. The required information is summarized in Table 2.1. 
Table 2.1. Forms, Diophantine equations, representations and distinct solutions for $R_{4}\left(t^{2}\right)$

\begin{tabular}{|c|l|c|c|c|l|}
\hline Form & Diophantine equation & $\begin{array}{c}\text { \# of } \\
\text { permutations }\end{array}$ & $\begin{array}{c}\text { \# of sign } \\
\text { changes }\end{array}$ & $\begin{array}{c}\text { total \# of } \\
\text { representations }\end{array}$ & $\begin{array}{c}\text { \# distinct } \\
\text { solutions }\end{array}$ \\
\hline$(w, x, y, z)$ & $w^{2}+x^{2}+y^{2}+z^{2}=t^{2}$ & 24 & 16 & 384 & $D_{4}\left(t^{2}\right)$ \\
\hline$(w, x, y, y)$ & $w^{2}+x^{2}+2 y^{2}=t^{2}$ & 12 & 16 & 192 & $D_{(1,1,2)}\left(t^{2}\right)$ \\
\hline$(w, x, x, x)$ & $w^{2}+3 x^{2}=t^{2}$ & 4 & 16 & 64 & $D_{(1,3)}\left(t^{2}\right)$ \\
\hline$(w, w, y, y)$ & $2 w^{2}+2 y^{2}=t^{2}$ & 6 & 16 & 96 & $D_{(2,2)}\left(t^{2}\right)$ \\
\hline$(0, x, y, z)$ & $x^{2}+y^{2}+z^{2}=t^{2}$ & 24 & 8 & 192 & $D_{3}\left(t^{2}\right)$ \\
\hline$(0, x, y, y)$ & $x^{2}+2 y^{2}=t^{2}$ & 12 & 8 & 96 & $D_{(1,2)}\left(t^{2}\right)$ \\
\hline$(0,0, y, z)$ & $y^{2}+z^{2}=t^{2}$ & 12 & 8 & 48 & $D_{2}\left(t^{2}\right)$ \\
\hline
\end{tabular}


By definition of the counting function $R_{4}\left(t^{2}\right)$, one has the identity

$$
\begin{aligned}
& 384 D_{4}\left(t^{2}\right)+192 D_{(1,1,2)}\left(t^{2}\right)+64 D_{(1,3)}\left(t^{2}\right)+96 D_{(2,2)}\left(t^{2}\right) \\
& +192 D_{3}\left(t^{2}\right)+96 D_{(1,2)}\left(t^{2}\right)+48 D_{2}\left(t^{2}\right)=R_{4}\left(t^{2}\right) .
\end{aligned}
$$

Additionally, by definition of the first 4 forms, one has the identity

$$
D_{4}\left(t^{2}\right)+D_{(1,1,2)}\left(t^{2}\right)+D_{(1,3)}\left(t^{2}\right)+D_{(2,2)}\left(t^{2}\right)=R_{4}^{d}\left(t^{2}\right) .
$$

On the other hand, denote by $R_{(1,1,2)}^{d}\left(t^{2}\right)$ the number of non-zero distinct primitive solutions of the Diophantine equation $w^{2}+x^{2}+2 y^{2}=t^{2}$, and by $R_{3}^{d}\left(t^{2}\right)$ the number of non-zero distinct primitive solutions of $x^{2}+y^{2}+z^{2}=t^{2}$. Since the distinct primitive solutions defining $R_{3}^{d}\left(t^{2}\right)$ are of the three different forms $(x, y, z),(x, y, y)$, and $(0, y, z)$, with distinct entries $x, y, z \neq 0$, one obtains similarly to (2.5) the identity

$$
D_{3}\left(t^{2}\right)+D_{(1,2)}\left(t^{2}\right)=R_{3}^{d}\left(t^{2}\right)
$$

To obtain an identity for $R_{(1,1,2)}^{d}\left(t^{2}\right)$, one considers similarly to Table 2.1 the different forms associated to the counting function denoted $R_{(1,1,2)}\left(t^{2}\right)$ (the number of primitive solutions of $x^{2}+y^{2}+2 z^{2}=t^{2}$ ) as summarized in Table 2.2. Though only partially required here, the whole information will be needed in Section 3. 
Table 2.2. Forms, Diophantine equations, representations and distinct solutions for $R_{(1,1,2)}\left(t^{2}\right)$

\begin{tabular}{|c|l|c|c|c|c|}
\hline Form & Diophantine equation & $\begin{array}{c}\text { \# of } \\
\text { permutations }\end{array}$ & $\begin{array}{c}\text { \# of sign } \\
\text { changes }\end{array}$ & $\begin{array}{c}\text { total \# of } \\
\text { representations }\end{array}$ & $\begin{array}{c}\text { \# distinct } \\
\text { solutions }\end{array}$ \\
\hline$(x, y, z)$ & $x^{2}+y^{2}+2 z^{2}=t^{2}$ & 2 & 8 & 16 & $D_{(1,1,2)}\left(t^{2}\right)$ \\
\hline$(x, y, y)$ & $x^{2}+3 y^{2}=t^{2}$ & 2 & 8 & 16 & $D_{(1,3)}\left(t^{2}\right)$ \\
\hline$(x, x, z)$ & $2 x^{2}+2 z^{2}=t^{2}$ & 1 & 8 & 8 & $D_{(2,2)}\left(t^{2}\right)$ \\
\hline$(z, z, x)$ & $2 z^{2}+2 x^{2}=t^{2}$ & 1 & 8 & 8 & $D_{(2,2)}\left(t^{2}\right)$ \\
\hline$(x, y, 0)$ & $x^{2}+y^{2}=t^{2}$ & 2 & 4 & 8 & $D_{2}\left(t^{2}\right)$ \\
\hline$(0, y, z)$ & $y^{2}+2 z^{2}=t^{2}$ & 2 & 4 & 8 & $D_{(1,2)}\left(t^{2}\right)$ \\
\hline
\end{tabular}


It is important to note that the forms $(x, x, z)$ and $(z, z, x)$, though solutions of the same Diophantine equation, generate distinct solutions of $x^{2}+y^{2}+2 z^{2}=t^{2}$. Therefore, by definition of the first 4 forms in Table 2.2 , one obtains the identity

$$
D_{(1,1,2)}\left(t^{2}\right)+D_{(1,3)}\left(t^{2}\right)+2 D_{(2,2)}\left(t^{2}\right)=R_{(1,1,2)}^{d}\left(t^{2}\right) .
$$

Now, solve (2.5)-(2.7) for $D_{4}\left(t^{2}\right), D_{3}\left(t^{2}\right)$, and $D_{(1,1,2)}\left(t^{2}\right)$, respectively, and insert into (2.4) to get after straightforward algebra the basic relationship

$$
\begin{aligned}
384 R_{4}^{d}\left(t^{2}\right)= & R_{4}\left(t^{2}\right)+192 R_{(1,1,2)}^{d}\left(t^{2}\right)-192 R_{3}^{d}\left(t^{2}\right)+128 D_{(1,3)}\left(t^{2}\right) \\
& -96 D_{(2,2)}\left(t^{2}\right)+96 D_{(1,2)}\left(t^{2}\right)-48 D_{2}\left(t^{2}\right)
\end{aligned}
$$

Once formulas for the right-hand side quantities have been determined, the left-hand side, which yields the number of primitive Pythagorean quintuples, will also be determined.

\section{Counting Formulas for the Ternary Squares Representations}

A formula for $R_{3}^{d}\left(t^{2}\right)$, which determines the number of primitive Pythagorean quadruples, is found in Hürlimann [5], Lemma 4. It is given by

$$
R_{3}^{d}\left(t^{2}\right)=\frac{1}{8} \cdot \varphi\left(t, \chi_{4}\right)-\frac{1}{2} D_{2}\left(t^{2}\right)+\frac{1}{2} D_{(1,2)}\left(t^{2}\right),
$$

where $t \geq 3$ is odd, and $\varphi\left(t, \chi_{4}\right)=t \cdot \prod_{p \mid t}\left(1-\chi_{4}(p) / p\right)$ is the twisted Euler (totient) function with Dirichlet character of period four defined by

$$
\chi_{4}(p)= \begin{cases}0, & p=2, \\ 1, & p \equiv 1 \quad(\bmod 4), \\ -1, & p \equiv 3 \quad(\bmod 4) .\end{cases}
$$


Applying the same algebraic counting mechanism as in Section 2, we derive now a formula for $R_{(1,1,2)}^{d}\left(t^{2}\right)$. From Table 2.2, one obtains similarly to (2.4) the relationship

$$
16 D_{(1,1,2)}\left(t^{2}\right)+16 D_{(1,3)}\left(t^{2}\right)+16 D_{(2,2)}\left(t^{2}\right)+8 D_{2}\left(t^{2}\right)+8 D_{(1,2)}\left(t^{2}\right)=R_{(1,1,2)}\left(t^{2}\right) .
$$

Solving (2.7) for $D_{(1,1,2)}\left(t^{2}\right)$ and inserting into (3.3), one obtains the formula

$$
16 R_{(1,1,2)}^{d}\left(t^{2}\right)=R_{(1,1,2)}\left(t^{2}\right)+16 D_{(2,2)}\left(t^{2}\right)-8 D_{2}\left(t^{2}\right)-8 D_{(1,2)}\left(t^{2}\right) .
$$

To obtain a formula for $R_{(1,1,2)}\left(t^{2}\right)$, one applies Möbius inversion to the counting formula for $r_{(1,1,2)}\left(t^{2}\right)$ by Cooper and Lam [3]. For the positive integer $t \geq 3$, let $t=2^{e_{2}} \cdot \prod_{i=1}^{m} p_{i}^{\lambda_{i}}$ be its unique prime factorization. Then, one has

$$
r_{(1,1,2)}\left(t^{2}\right)=4 b\left(e_{2}\right) \cdot \prod_{i=1}^{m}\left\{\frac{p_{i}^{\lambda_{i}+1}-1}{p_{i}-1}-\left(\frac{-2}{p_{i}}\right) \frac{p_{i}^{\lambda_{i}}-1}{p_{i}-1}\right\},
$$

where

$$
b\left(e_{2}\right)= \begin{cases}1, & \text { if } e_{2}=0 \\ 3, & \text { if } e_{2} \geq 1\end{cases}
$$

and the values of the Legendre symbol are given by

$$
\left(\frac{-2}{p}\right)= \begin{cases}1, & \text { if } p \equiv 1 \text { or } 3(\bmod 8) \\ -1, & \text { if } p \equiv 5 \text { or } 7 \quad(\bmod 8)\end{cases}
$$

From this, one gets through application of the Möbius inversion formula (2.3), the following formula for the number of primitive solutions of the equation $x^{2}+y^{2}+2 z^{2}=t^{2}$ : 


$$
R_{(1,1,2)}\left(t^{2}\right)= \begin{cases}4 \cdot \prod_{i=1}^{m} p_{i}^{\lambda_{i}-1}\left\{p_{i}-\left(\frac{-2}{p_{i}}\right)\right\}=4 \cdot \varphi\left(t, \chi_{8}\right), \quad \text { if } t \equiv 1,2,3(\bmod 4), \\ 0, \text { if } t \equiv 0(\bmod 4)\end{cases}
$$

where $\varphi\left(t, \chi_{8}\right)=t \cdot \prod_{p \mid t}\left(1-\chi_{8}(p) / p\right)$ is the twisted Euler (totient) function with Dirichlet character of period eight defined by

$$
\chi_{8}(p)= \begin{cases}1, & \text { if } p \equiv 1 \text { or } 3(\bmod 8) \\ -1, & \text { if } p \equiv 5 \text { or } 7 \quad(\bmod 8)\end{cases}
$$

We are ready for the following result.

Proposition 3.1 (Number of non-trivial distinct representations of squares by the ternary quadratic form $\left.x^{2}+y^{2}+2 z^{2}=t^{2}\right)$. One has the formula

$$
R_{(1,1,2)}^{d}\left(t^{2}\right)=\left\{\begin{array}{l}
\frac{1}{4} \varphi\left(t, \chi_{8}\right)-\frac{1}{2} D_{2}\left(t^{2}\right)-\frac{1}{2} D_{(1,2)}\left(t^{2}\right), \quad \text { if } t>2 \text { is odd }, \\
\frac{1}{2} \varphi\left(s, \chi_{8}\right)+D_{2}\left(s^{2}\right), \quad \text { if } t=2 s, s>1 \text { odd }, \\
1, \quad \text { if } t=2, \\
0, \quad \text { if } 4 \mid t .
\end{array}\right.
$$

Proof. This follows from (3.4) using (3.8). If $t>2$ is odd, then one has $D_{(2,2)}\left(t^{2}\right)=0$ because the equation $2 x^{2}+2 y^{2}=t^{2}$ has no solution. If $t=2 s$ with $s>1$ odd, one sees that

$$
D_{(2,2)}\left(t^{2}\right)=D_{2}\left(2 s^{2}\right)=D_{2}\left(s^{2}\right)
$$

Moreover, one has $D_{2}\left(4 s^{2}\right)=0$ (see Cooper and Hirschhorn [2], Theorem 1, Equation (1.6)) and $D_{(1,2)}\left(4 s^{2}\right)=0$. The latter is seen as follows. If 
$x^{2}+2 y^{2}=4 s^{2}$, then necessarily $x=2 X$, hence $2 X^{2}+y^{2}=2 s^{2}$ and $2 \mid y$. But then 2| $\operatorname{gcd}(x, y, t)$, which contradicts the condition $\operatorname{gcd}(x, y, t)=1$. Similarly, if $4 \mid t$, then all terms in the right-hand side of (3.4) vanish. The result is shown.

\section{Counting Formulas for the Binary Squares Representations}

According to the basic relationship (2.8) formulas for the number of binary squares representations $D_{2}\left(t^{2}\right), D_{(1,2)}\left(t^{2}\right), D_{(2,2)}\left(t^{2}\right)$, and $D_{(1,3)}\left(t^{2}\right)$ must be determined. Again, we suppose that the positive integer $t \geq 2$ has the prime factorization $t=2^{e_{2}} \cdot \prod_{i=1}^{m} p_{i}^{\lambda_{i}}$. The first two counting functions are well-known and given by

$$
D_{2}\left(t^{2}\right)= \begin{cases}2^{m-1}, & \text { if } p_{i} \equiv 1(\bmod 4), \quad i=1, \ldots, m, e_{2}=0, \\ 0, & \text { otherwise }\end{cases}
$$

respectively,

$$
D_{(1,2)}\left(t^{2}\right)= \begin{cases}2^{m-1}, & \text { if } p_{i} \equiv 1,3(\bmod 8), \quad i=1, \ldots, m, e_{2}=0 \\ 0, & \text { otherwise }\end{cases}
$$

A reduction formula for $D_{(2,2)}\left(t^{2}\right)$ is contained in the proof of Proposition 3.1. One has

$$
D_{(2,2)}\left(t^{2}\right)=\left\{\begin{array}{l}
D_{2}\left(s^{2}\right), \text { if } t=2 s, s \text { odd } \\
0, \text { otherwise }
\end{array}\right.
$$

It remains to determine the number of distinct primitive solutions of the equation $x^{2}+3 y^{2}=t^{2}$. Again, one first finds a formula for the number of all representations $r_{(1,3)}\left(t^{2}\right)$ taking into account zeros, permutations, and sign changes, and then apply Möbius inversion to get an expression 
for $R_{(1,3)}\left(t^{2}\right)$, from which one gets $D_{(1,3)}\left(t^{2}\right)=\frac{1}{4} R_{(1,3)}\left(t^{2}\right)$. According to Dickson [4], Exercises XXII, no. 3, p. 80, Section 51 (see also Berndt [1], Theorem 3.7.5, p. 75), one has for an odd number $s$ :

$$
r_{(1,3)}\left(2^{k} s\right)=\left\{\begin{array}{l}
2 E(s), \quad \text { if } k=0 \\
6 E(s), \quad \text { if } k \geq 2 \text { is even, } \\
0, \quad \text { otherwise, }
\end{array}\right.
$$

where $E(n)=d_{1,3}(n)-d_{2,3}(n)$ is the excess of the number of divisors $\equiv 1(\bmod 3)$ of $n$ over the number of divisors $\equiv 2(\bmod 3)$ of $n$. Based on the prime factorization $t=2^{e_{2}} \cdot \prod_{i=1}^{m} p_{i}^{\lambda_{i}}$, one obtains after some calculation the formula

$$
D_{(1,3)}\left(t^{2}\right)= \begin{cases}2^{m-1}, & \text { if } p_{i} \equiv 1(\bmod 3), \quad i=1, \ldots, m, e_{2}=0, \\ 2^{m}, & \text { if } p_{i} \equiv 1(\bmod 3), \quad i=1, \ldots, m, e_{2}=1, \\ 0, & \text { otherwise. }\end{cases}
$$

\section{The Exact Number of Primitive Pythagorean Quintuples}

Up to the counting function $R_{4}\left(t^{2}\right)$, all terms on the right-hand side of the basic relationship (2.8) have been determined in the Sections 3 and 4. For the remaining term, one borrows from Cooper and Hirschhorn [2], Theorem 1, Equation (1.7), the expression

$$
R_{4}\left(t^{2}\right)= \begin{cases}8 t^{2} \cdot \prod_{p \mid t}(1+1 / p), & \text { if } t \text { is odd }, \\ 16 s^{2} \cdot \prod_{\substack{p \mid s \\ \text { otherwise. }}}(1+1 / p), & \text { if } t=2 s, s \text { odd } \\ 0, & \end{cases}
$$

The main result of the present contribution follows. 
Theorem 5.1. The number of distinct primitive Pythagorean quintuples is determined as follows:

$$
\begin{aligned}
& R_{4}^{d}\left(t^{2}\right)=\left\{\begin{array}{l}
\frac{1}{48} t^{2} \cdot \prod_{p \mid t}(1+1 / p)-\frac{1}{16} \varphi\left(t, \chi_{4}\right)+\frac{1}{8} \varphi\left(t, \chi_{8}\right) \\
-\frac{1}{8} D_{2}\left(t^{2}\right)-\frac{1}{4} D_{(1,2)}\left(t^{2}\right)+\frac{1}{3} D_{(1,3)}\left(t^{2}\right)
\end{array}\right\}, \text { if } t>2 \text { is odd, } \\
& R_{4}^{d}\left(t^{2}\right)=\left\{\frac{1}{24} s^{2} \cdot \prod_{p \mid s}(1+1 / p)+\frac{1}{4} \varphi\left(s, \chi_{8}\right)+\frac{1}{4} D_{2}\left(s^{2}\right)+\frac{1}{3} D_{(1,3)}\left(4 s^{2}\right)\right\}, \\
& \text { if } t=2 s, s>1 \text { odd, } \\
& R_{4}^{d}\left(t^{2}\right)=\left\{\begin{array}{lll}
1, & \text { if } & t=2 \\
0, & \text { if } & 4 \mid t
\end{array}\right\} .
\end{aligned}
$$

Proof. We distinguish between the two main cases.

Case 1. $t>2$ is odd.

Since $D_{(2,2)}\left(t^{2}\right)=0$ by (4.3), one gets through insertion of (3.1) and (3.10) into (2.8) that

$$
\begin{aligned}
384 R_{4}^{d}\left(t^{2}\right)= & R_{4}\left(t^{2}\right)+192\left\{\frac{1}{4} \varphi\left(t, \chi_{8}\right)-\frac{1}{2} D_{2}\left(t^{2}\right)-\frac{1}{2} D_{(1,2)}\left(t^{2}\right)\right\} \\
& -192\left\{\frac{1}{8} \cdot \varphi\left(t, \chi_{4}\right)-\frac{1}{2} D_{2}\left(t^{2}\right)+\frac{1}{2} D_{(1,2)}\left(t^{2}\right)\right\} \\
& +128 D_{(1,3)}\left(t^{2}\right)+96 D_{(1,2)}\left(t^{2}\right)-48 D_{2}\left(t^{2}\right) \\
= & R_{4}\left(t^{2}\right)-24 \varphi\left(t, \chi_{4}\right)+48 \varphi\left(t, \chi_{8}\right)-48 D_{2}\left(t^{2}\right) \\
& -96 D_{(1,2)}\left(t^{2}\right)+128 D_{(1,3)}\left(t^{2}\right) .
\end{aligned}
$$

Inserting (5.1) and dividing by 384 one obtains (5.2). 
Case 2. $t=2 s, s>1$ odd.

In this situation, one has $R_{3}^{d}\left(t^{2}\right)=0$ (no primitive cuboid with even diagonal), as well as $D_{2}\left(t^{2}\right)=D_{(1,2)}\left(t^{2}\right)=0$ and $D_{(2,2)}\left(t^{2}\right)=D_{2}\left(s^{2}\right)$ by (4.1)-(4.3). Inserting this and (3.10) into (2.8), one has

$$
\begin{aligned}
384 R_{4}^{d}\left(t^{2}\right) & =R_{4}\left(t^{2}\right)+192\left\{\frac{1}{2} \varphi\left(s, \chi_{8}\right)+D_{2}\left(s^{2}\right)\right\}+128 D_{(1,3)}\left(t^{2}\right)-96 D_{2}\left(s^{2}\right) \\
& =R_{4}\left(t^{2}\right)+96 \varphi\left(s, \chi_{8}\right)+96 D_{2}\left(s^{2}\right)+128 D_{(1,3)}\left(t^{2}\right) .
\end{aligned}
$$

Inserting (5.1) and dividing by 384 one obtains (5.2).

The exact calculation of all terms in (5.2)-(5.3) requires a factorization table of the distinct prime factors of all odd numbers. To illustrate, we have calculated the number of primitive Pythagorean quintuples for all $t<1000$. Table 5.1 provides the detailed cumulative count for all $t<100$ and Table 5.2 a summarized count. 
Table 5.1. Cumulative number of primitive Pythagorean quintuples below 100

\begin{tabular}{|cc|cc|cc|cc|cc|cc|}
\hline Odd $t$ & Cum. & Odd $t$ & Cum. & Odd $t$ & Cum. & Odd $t$ & Cum. & Even $t$ & Cum. & Even $t$ & Cum. \\
\hline 3 & 0 & 29 & 122 & 53 & 693 & 77 & 2094 & 2 & 1 & 54 & 237 \\
5 & 1 & 31 & 145 & 55 & 780 & 79 & 2231 & 6 & 2 & 58 & 281 \\
7 & 3 & 33 & 177 & 57 & 874 & 81 & 2413 & 10 & 5 & 62 & 331 \\
9 & 5 & 35 & 216 & 59 & 951 & 83 & 2563 & 14 & 10 & 66 & 402 \\
11 & 8 & 37 & 248 & 61 & 1034 & 85 & 2762 & 18 & 16 & 70 & 484 \\
13 & 13 & 39 & 294 & 63 & 1160 & 87 & 2980 & 22 & 24 & 74 & 553 \\
15 & 21 & 41 & 332 & 65 & 1281 & 89 & 3152 & 26 & 36 & 78 & 651 \\
17 & 28 & 43 & 374 & 67 & 1380 & 91 & 3373 & 30 & 54 & 82 & 733 \\
19 & 37 & 45 & 443 & 69 & 1518 & 93 & 3621 & 34 & 71 & 86 & 823 \\
21 & 51 & 47 & 493 & 71 & 1629 & 95 & 3867 & 38 & 92 & 90 & 967 \\
23 & 64 & 49 & 554 & 73 & 1746 & 97 & 4071 & 42 & 124 & 94 & 1073 \\
25 & 82 & 51 & 630 & 75 & 1936 & 99 & 4366 & 46 & 153 & 98 & 1202 \\
27 & 102 & & & & & & & 50 & 192 & & \\
\hline
\end{tabular}


Table 5.2. Cumulative number of primitive Pythagorean quintuples below 1000

\begin{tabular}{|c|c|c|c|}
\hline Limit & Odd $t$ & Even $t=2 s$ & Total \\
\hline 100 & 4366 & 1202 & 5568 \\
200 & 34142 & 9096 & 43238 \\
300 & 115170 & 29870 & 145040 \\
400 & 272740 & 69681 & 342421 \\
500 & 530798 & 135812 & 666610 \\
600 & 916248 & 233467 & 1149715 \\
700 & 1455119 & 368486 & 1823605 \\
800 & 2168116 & 551028 & 2719144 \\
900 & 3087846 & 780450 & 3868296 \\
1000 & 4232085 & 1070218 & 5302303 \\
\hline
\end{tabular}

\section{References}

[1] B. C. Berndt, Number Theory in the Spirit of Ramanujan, Student Mathematical Library, Vol. 34, American Mathematical Society, Providence, RI, 2006.

[2] S. Cooper and M. D. Hirschhorn, On the number of primitive representations of integers as sum of squares, Ramanujan Journal 13 (2007), 7-25.

[3] S. Cooper and H. Y. Lam, On the Diophantine equation $n^{2}=x^{2}+b y^{2}+c z^{2}$, Journal of Number Theory 133 (2013), 719-737.

[4] L. E. Dickson, Introduction to the Theory of Numbers, University of Chicago Press, Reprint (1957), Dover Publications, 1929.

[5] W. Hürlimann, Exact and asymptotic evaluation of the number of distinct primitive cuboids, Journal of Integer Sequences 18(2), Article 15.2.5. (2015).

[6] E. Grosswald, Representations of Integers as Sums of Squares, Springer, New York, 1985.

[7] J. L. Lagrange, Démonstration d'un théorème d'arithmétique, Nouveaux Mémoires de l'Acad. Royale des Sciences et Belles Lettres de Berlin, (Oeuvres 3 (1770), 695-795). 